\title{
Transient corneal changes associated with the use of gold foil electrodes
}

\author{
G W AYLWARD, $K$ A McClellan, R THOMAS, AND F A BILlson \\ From the Department of Ophthalmology, University of Sydney, Sydney Eye Hospital, Woolloomooloo, \\ 2011, Australia
}

SUMMARY The corneas of 50 normal subjects were examined before and after electroretinography performed with gold foil electrodes. Examination included slit-lamp biomicroscopy and staining with sodium fluorescein. All corneas were normal on examination prior to electroretinography. Three types of transient corneal changes were observed - punctate epithelial keratitis, corneal erosions, and stromal thinning. Each cornea was assigned a numerical damage score based on a simple scoring system. Thirty one subjects $(62 \%)$ had some degree of corneal change, and in three cases $(6 \%)$ follow-up was required. Multiple regression analysis was performed to discover any risk factors. Both age of the subject and the use of local anaesthetic were strongly associated with corneal changes.

The use of electroretinography as a clinically useful investigation was facilitated by the introduction of corneal contact lens electrodes. ${ }^{2}$ These allowed reliable and reproducible recordings with a stable baseline. Several improvements and refinements to their basic design were made over the next three decades. ${ }^{3}$ However, such electrodes caused discomfort to the subject and trauma to the corneal epithelium. ${ }^{+}$This restricted their use in children and in patients with pre-existing corneal disease. A further disadvantage was interference with the optics of the eye, which became important with the introduction of patterned stimuli. The use of foil electrodes $^{5 t}$ and other non-contact lens electrodes' solved many of these problems and enabled longer recording sessions."

We have used gold foil electrodes in our department for both routine electroretinography and research purposes. It has come to our attention that a small proportion of patients suffer transient symptoms after removal of the electrodes and that these symptoms are associated with corneal changes which are visible on slit-lamp biomicroscopy. The symptoms include blurred vision, ocular discomfort, and watering, which occasionally persist for several Correspondence to $\mathrm{Dr}$ G W Aylward, 8 Kinburn Street, Rotherhithe, London SE16 1DN. days. We have also observed punctate epithelial keratitis (PEK), areas of corneal erosion, and patches of stromal thinning. This paper describes the corneal changes in a consecutive series of normal subjects undergoing electroretinography with gold foil electrodes.

\section{Patients and methods}

The subjects participating in this study were a group of normal volunteers undergoing a new ERG protocol which we are developing. ${ }^{8}$ Fifty consecutive normal subjects were included. There were 21 males and 29 females and the average age was 38.6 years (range 20-57). A full medical and ophthalmological history was taken from each subject. Examination included corrected visual acuity, measurement of intraocular pressure, and slit-lamp biomicroscopy, both before and after staining with fluorescein. The pupils were dilated with one drop of tropicamide in each eye, and the subjects were then dark adapted for 30 minutes. At the end of this period gold foil electrodes were inserted under dim red illumination. We used electrodes manufactured in our own laboratory from $19 \mu \mathrm{m}$ thick gold-plated plastic and electrically conducting adhesive tape. ${ }^{9}$ These electrodes are $20 \mathrm{~mm}$ long and $5 \mathrm{~mm}$ wide and are taped 


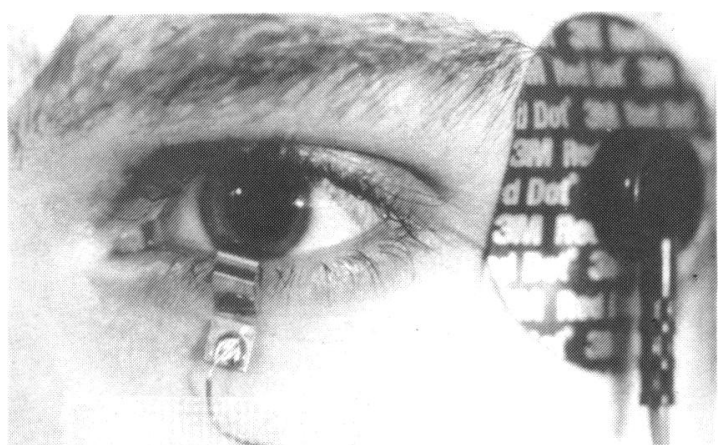

Fig. 1 Gold foil electrode shown in position. The temple reference and forehead earth electrodes are silver/silver chloride types.

to the lower eye lid so that they lie centrally on the cornea (Fig. 1). A local anaesthetic was not used routinely but was offered to all subjects and given if requested or if excessive blinking occurred. If required, one drop of proxymetacaine $0.5 \%$ was instilled into each eye.

Multiple ERGs to Ganzfeld stimuli were then recorded over a period of approximately 20 minutes. The intensity of the stimulus was varied by means of neutral density filters from psychophysical threshold to the maximum brightness available from the Grass PS22 photic stimulator. Amplified signals were averaged with computer software with automatic artefact rejection. The number of artefact-free traces required was 32 for low intensity stimuli and 16 for high intensity stimuli. Further details of the electroretinography will not be given here, as they are not directly relevant to the present study. When recordings were completed the electrodes were removed and subjects were examined by slit-lamp biomicroscopy. The presence or absence of limbal injection was noted, and each cornea was graded for the presence of three lesions by means of a simple grading scheme (Table 1). A total score was assigned to each subject, being the sum of the scores for each eye. Thirty minutes after removal of the electrodes the subjects were asked about ocular symptoms, particularly discomfort and watering. They were then allowed to leave and were asked to report back if symptoms persisted.

\section{Results}

No abnormalities on slit-lamp biomicroscopy were noted in any of the subjects prior to insertion of the electrodes. Nine subjects $(18 \%)$ requested the application of local anaesthetic. Thirty minutes after removal of the electrodes eight subjects $(16 \%)$ complained of some ocular symptoms. The frequen-
Table 1 Scoring system used to quantify corneal changes. Each cornea was assigned a score for each of the lesions shown. The total corneal score for each subject was the sum of all scores for both eyes (maximum 14)

\begin{tabular}{|c|c|c|c|}
\hline \multirow[b]{2}{*}{ Lesion } & \multicolumn{3}{|l|}{ Score } \\
\hline & 0 & 1 & 2 \\
\hline Limbal injection & Absent & Present & - \\
\hline $\begin{array}{l}\text { Punctate } \\
\text { epithelial } \\
\text { keratitis }\end{array}$ & Absent & $\begin{array}{l}\text { Less than } 1 / 4 \text { of } \\
\text { corneal area } \\
\text { involved }\end{array}$ & $\begin{array}{l}\text { More than } 1 / 4 \text { of } \\
\text { corneal area } \\
\text { involved }\end{array}$ \\
\hline $\begin{array}{l}\text { Corneal } \\
\text { erosion }\end{array}$ & Absent & $\begin{array}{l}\text { One lesion less } \\
\text { than } 1 \mathrm{~mm} \text { in } \\
\text { diameter }\end{array}$ & $\begin{array}{l}\text { More than one } \\
\text { lesion, or one } \\
\text { lesion greater } \\
\text { than } 1 \mathrm{~mm} \text { in } \\
\text { diameter }\end{array}$ \\
\hline $\begin{array}{l}\text { Stromal } \\
\text { thinning }\end{array}$ & Absent & $\begin{array}{l}\text { One lesion less } \\
\text { than } 1 / 3 \text { stromal } \\
\text { thinning }\end{array}$ & $\begin{array}{l}\text { More than one } \\
\text { lesion, or one } \\
\text { lesion greater than } \\
1 / 3 \text { stromal } \\
\text { thinning }\end{array}$ \\
\hline
\end{tabular}

cies of limbal injection, PEK, corneal erosion, and corneal thinning are shown in Fig. 2. The proportion of subjects who had a corneal sign present in at least one eye was $31(62 \%)$. This may be subdivided into $17(34 \%)$ for corneal injection, $31(62 \%)$ for PEK, $10(20 \%)$ for corneal erosion, and $8(16 \%)$ for

Number of

Subjects

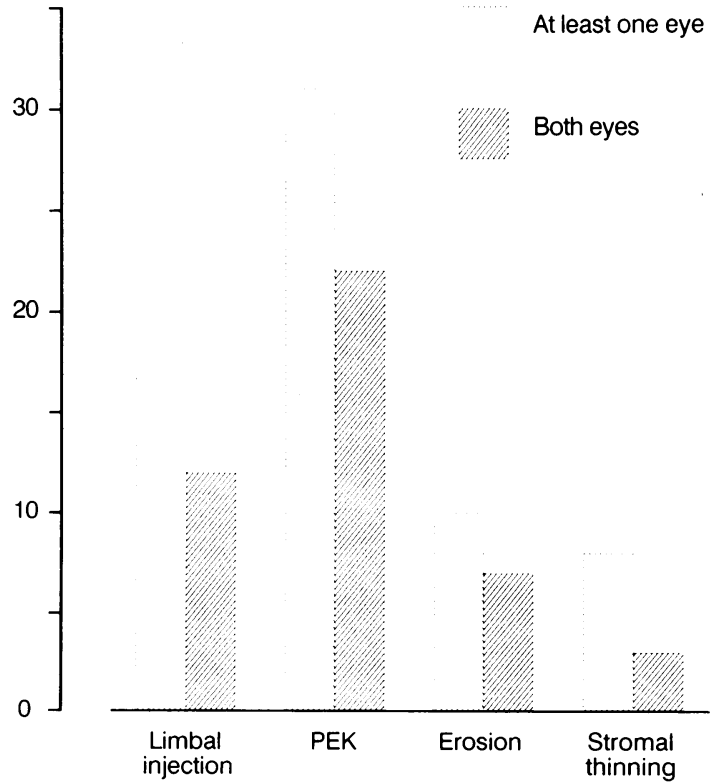

Fig. 2 Frequencies of limbal injection, punctate epithelial keratitis, and stromal thinning. Frequencies are shown for involvement of at least one eye and for involvement of both eyes. 


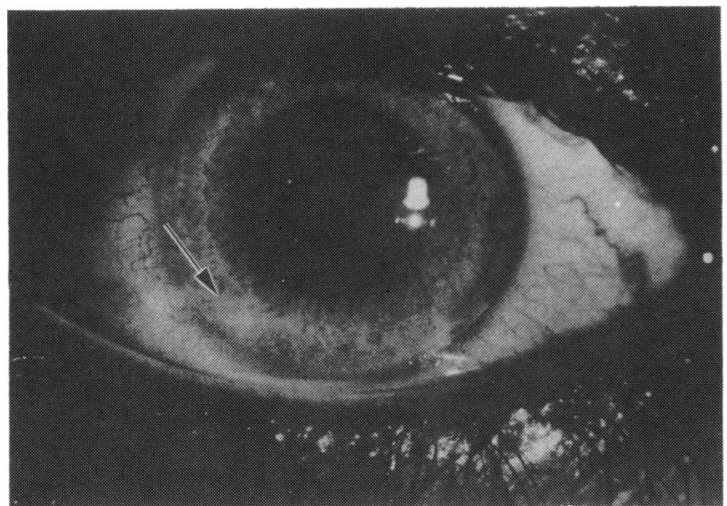

Fig. 3a Slit-lamp photograph of cornea stained with fluorescein. This subject was 21 years old but had not been given local anaesthetic. Note the area of punctate epithelial keratitis lying just nasal to the position where the electrode was placed (arrow).

corneal thinning. Examples of PEK, erosion, and thinning are shown in Figs. 3A, B, C. The affected areas tended to be adjacent to the vertical edge of the electrode. A scatter plot of the total corneal score plotted against the age of the subject is shown in Fig. 4. The relationship between the total corneal score, the use of local anaesthetic, and the subject's age and sex was examined by multiple regression analysis. The total corneal score followed a skewed deviation, so a square root transformation was used to decrease errors which might arise owing to departure from normality. Regression analysis on the

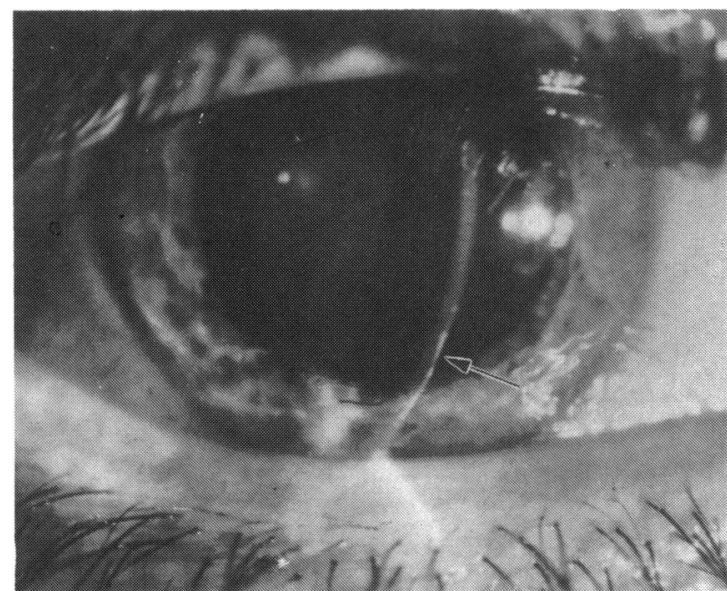

Fig. 3c Slit-lamp photograph of cornea stained with fluorescein. This subject was 51 years old and had been given local anaesthetic. Note the area of localised stromal thinning (arrow). Thirty minutes earlier the thinning was much more marked.

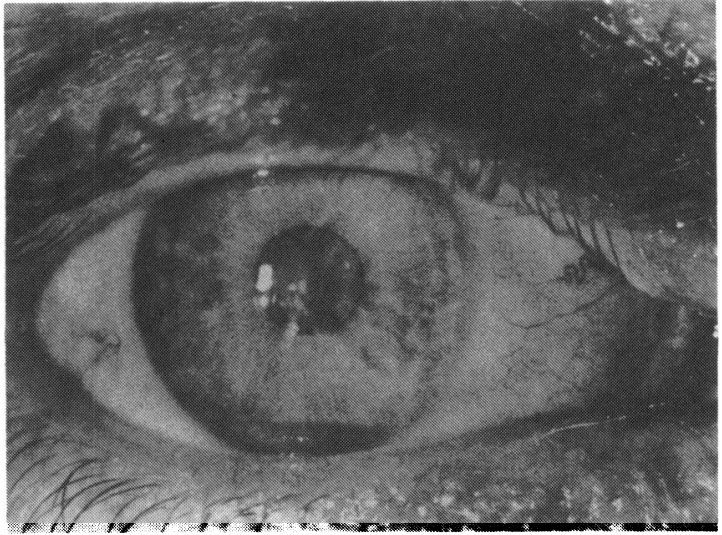

Fig. 3b Slit-lamp photograph of cornea stained with fluorescein. This subject was 27 years old and had been given local anaesthetic. Note widespread areas of corneal erosion.

transformed data revealed a significant correlation between total corneal score and two independent variables, age and use of local anaesthetic. Both these correlations were significant at the $p<0 \cdot 01$ level. There was no relationship between total corneal score and the subject's sex.

Three subjects (aged 44, 55, and 57, local anaesthetic used in all three) had symptoms which were severe enough for them to return the following day. These subjects had total corneal scores of 10,11 , and 12. Slit-lamp examination revealed persistent PEK,
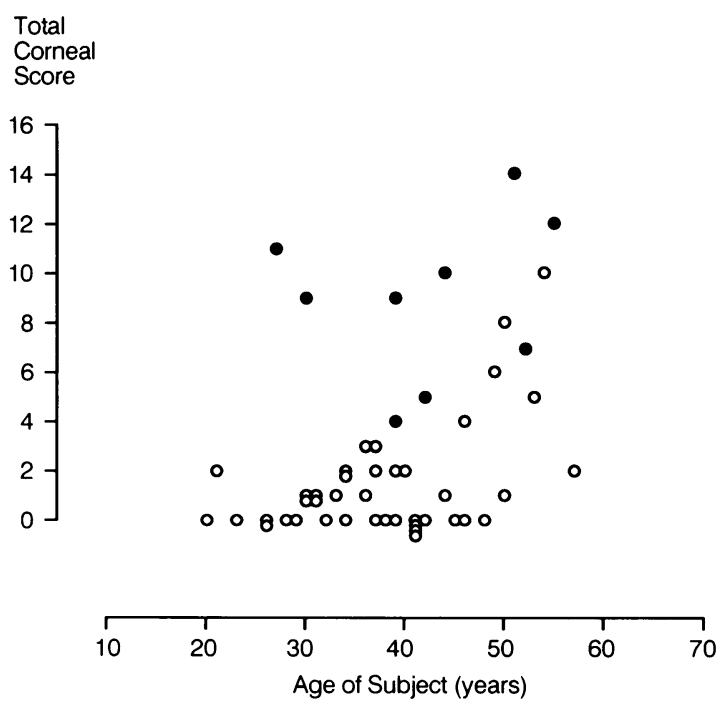

Fig. 4 Total corneal score plotted against age for subjects who were not given anaesthetic (open circles) and subjects who were given anaesthetic (filled circles). 
though the other changes which were present the day before had vanished. One other subject (aged 51, local anaesthetic used) with a total corneal score of 14 agreed to remain for photography. At the examination following the ERG corneal thinning was present in both eyes, and in one area the thinning was marked, the corneal thickness being one-third of normal in the affected area. Anterior segment photography was performed 30 minutes later, by which time the thinning had reduced (Fig. 3C). One hour later there was no visible thinning, though erosion and PEK remained. The next day he had no symptoms and all the corneal signs had resolved.

\section{Discussion}

The present study indicates that transient corneal changes are common following the use of gold foil electrodes. The proportion of normal subjects with detectable corneal changes in at least one eye was $62 \%$. In eight subjects these were associated with the presence of symptoms, and in three these were severe enough to warrant a return visit the following day. However, all changes were transient, and there were no serious sequelae in any of the subjects or in the several hundred patients who have had electroretinography in our department over the last few years.

There was significant association between the severity of the corneal changes and two factors; the age of the subject and the use of anaesthetic. The relationship with age is probably explained by changes in the composition of the tear film. ." Local anaesthetic interrupts the afferent pathway and reduces reflex lacrimation. It may also reduce the frequency of blinking, a factor which could well be exacerbated by the absence of a formed visual image in the Ganzfeld stimulator. Local anaesthetic can be toxic to the epithelium, ${ }^{11}$ but it is unlikely that one drop of proxymetacaine alone could cause the extensive changes observed in this study. Furthermore, of the 31 subjects with some degree of corneal change, 22 had not received local anaesthetic. A weakness of the study is that the use of anaesthetic was decided by the subject and not according to a randomised protocol. It may be that patients requiring anaesthetic have an additional factor leading to corneal changes, such as excessive blinking.

Punctate epithelial keratitis is a non-specific appearance seen in association with a variety of insults to the cornea. Its appearance in this context is most likely related to trauma from the electrode. It is noteworthy that the concentration of PEK was highest near the vertical edges of the electrode. Our electrodes are slightly thicker at $19 \mu \mathrm{m}$ than the $12 \mu \mathrm{m}$ gold plated Mylar film used by Arden et al.$^{\circ}$ It is also possible that the edges of our electrodes are not as smooth as commercially available electrodes. However, a visual inspection under a binocular microscope $(\times 50)$ revealed no obvious difference between the two. The presence of small corneal erosions is probably also related to trauma. Erosions were present in all eight of the subjects who reported symptoms 30 minutes after removal of the electrodes, and they are the most likely cause of the discomfort. There is a potential risk of corneal infection in the presence of a breach in the epithelial barrier, though we know of no reported cases of infectious keratitis following electroretinography with gold foil electrodes. It may be prudent, however, to administer topical antibiotic to anyone with an erosion. In the light of this the sterility of the electrodes becomes important. Commercially available gold foil electrodes are expensive and tend to be reused, though they are difficult to sterilise. The electrodes used in this study are very cheap and quick to make and can therefore be treated as disposable. This is now our our routine practice.

Eight of the subjects in this study had some degree of corneal thinning. This too was commonly seen in the areas adjacent to the vertical edges of the electrodes. This lesion was very similar to dellen'2 and probably arose through the similar mechanisms. There is evidence to suggest that dellen arise owing to a local deficiency in the tear film ${ }^{13}$ and may be exacerbated by a reduction in blinking. ${ }^{1+}$ The rapid disappearance of the thinning in one of our subjects over the course of an hour was striking and tends to support the notion that it was related to a temporary disturbance in the tear film.

In summary, we have found a high incidence of transient corneal changes following the use of gold foil electrodes for electroretinography. These changes were associated with the subject's age and the use of local anaesthetic. The following recommendations are made. (1) Local anaesthetic should be used with gold foil electrodes only when absolutely necessary. (2) Patients should undergo slit-lamp biomicroscopy following electroretinography, and if any erosions are present treatment with topical antibiotic should be instituted. (3) Attention should be given to the sterility of the electrodes, and if possible they should be discarded after a single use.

This work was supported by the Sydney Eye Hospital Foundation (Sylvia Kane Bequest for Research into Diabetic Retinopathy) and by Diabetes Australia (Lions Diabetic Retinopathy Research Grant).

The authors thank the following for their help: Professor G Berry for advice on statistical method; Mr Brett Jeffrey for computer programming and assistance with electroretinography: $\mathrm{Mr}$ James Borlaise for photographic processing; and all study participants for their goodwill and co-operation. 


\section{References}

1 Riggs LA. Continuous and reproducible records of the electrical activity of the human retina. Proc Soc Exp Biol Med 1941; 48: 204-7.

2 Karpe G. The basis of clinical electroretinography. Acta Ophthalmol (Kbh) 1945; 24 (suppl): 1-118.

3 Sundmark E. ERG recording with different types of contact glass. Acta Ophthalmol (Kbh) 1962; 70 (suppl): 62-9.

4 Zisman F. A comparison of corneal trauma induced by two techniques for performing electroretinography. Am J Optom Physiol Opt 1974; 51: 978-81.

5 Borda RP, Gillam RM, Coats AC. Gold-coated Mylar (GMC) electrode for electroretinography. Doc Ophthalmol Proc Ser 1977; 15: 339-43.

6 Arden GB, Carter RM, Hogg C, Siegel IM, Margolis S. A gold foil electrode: extending the horizons for clinical electroretinography. Invest Ophthalmol Vis Sci 1979; 18: 421-6.

7 Dawson WW, Trick GL, Litzkow CA. Improved electrode for electroretinography. Invest Ophthalmol Vis Sci 1979; 18: 988-91

8 Aylward GW, Vaegan, Billson FA. The scotopic threshold response in man. Clin Vis Sci in press.

9 Vaegan. An improved method of constructing pattern electroretinogram electrodes. Doc Ophthalmol Proc Ser 1984: 40: 287-91.

10 Farris RL, Stuchell RN, Mandel ID. Basal and reflex human tear analysis. Ophthalmology 1981: 88: 853-61.

11 vd Eerden AAJJ. Changes in corneal epithelium due to local anaesthetics. Ophthalmologica 1962; 143: 154-62.

12 Fuchs E. Über Dellen in der Hornhaut. Graefes Arch Clin Exp Ophthalmol 1911; 78: 82-92.

13 Trantas N. On two cases of local intense and transitory corneal dehydration. Bull Soc Hellen Ophthalmol 1952; 20: 163-70.

14 Mackie IA. Localized corneal drying in association with dellen. pterygia and related lesions. Trans Ophthalmol Soc UK 1971: 91: $129-45$.

Accepted for publication 25 May 1989. 